EPJ Web of Conferences 81, 04011 (2014)

DOI: 10.1051/epjconf/ 20148104011

(C) Owned by the authors, published by EDP Sciences, 2014

\title{
Measurement of the Polarised Drell-Yan process at COMPASS
}

\author{
Márcia Quaresma ${ }^{1, a}$ \\ for the COMPASS Collaboration \\ ${ }^{1}$ LIP - Laboratório de Instrumentação e Física Experimental de Partículas, Av. Elias Garcia 14-1, 1000-149 \\ Lisboa, Portugal
}

\begin{abstract}
The COMPASS experiment at CERN has been playing an important role in the studies of the spin content of the nucleon. The Semi-Inclusive Deep Inelastic Scattering (SIDIS) process gives access to the transverse momentum dependent parton distribution functions (TMDs) by the measurement of azimuthal asymmetries that have been studied in COMPASS and published in recent years. TMDs are also accessible by the transversely polarised Drell-Yan (DY) process which will be measured in COMPASS. This will be the first ever polarised DY measurement. The valence quarks region will be dominant due to the use of a negative pion beam at $190 \mathrm{GeV} / c$ momentum impinging on a transversely polarised ammonia target. The QCD prediction that Sivers TMD change sign when accessed by SIDIS or by DY will be checked by the COMPASS measurement. The data taking is scheduled to start in the fall of this year. After one year of data collection, a statistical error below $2 \%$ in the azimuthal asymmetry related to the $u$ quark Sivers function is expected. Details of the final experimental setup will be presented.
\end{abstract}

\section{Introduction}

The nucleon structure in QCD leading twist, taking into account the intrinsic transverse momentum of the quarks $k_{T}$, is described by 8 transverse momentum dependent parton distribution functions (TMD PDFs) for each quark flavour. Both the single polarised Drell-Yan measurement and the transversely polarised SIDIS give access to them. The DY process is considered an excellent tool to access TMD PDFs. Contrary to SIDIS, in the DY cross-section there is no fragmentation functions involved but only the convolution of PDFs. In addition, all the TMD PDFs are expected to be sizeable in the valence $u$ quark region, which is dominant when a negative pion beam is used and, moreover, the QCD TMD approach is valid in the region $Q\left(M_{\mu \mu}>4 \mathrm{GeV} / c^{2}\right) \gg\left\langle p_{T}\right\rangle \sim 1 \mathrm{GeV} / c$.

The single transversely polarised DY cross-section in LO can be written as a sum of 4 angular modulations. Each angular modulation has an amplitude that contains the convolution of two TMD PDFs. These amplitudes are accessed via the measurement of the angular azimuthal asymmetries between the two oppositely transversely polarised target cells. The asymmetry $A_{U}^{\cos 2 \phi}$ relates to the beam quark Boer-Mulders function $h_{1}^{\perp}$ convoluted with the target quark Boer-Mulders one $h_{1}^{\perp} . A_{T}^{\sin \phi_{S}}$ relates to the beam quark unpolarised PDF $f_{1}$ and the target quark Sivers function $f_{1 T}^{\perp}$. $A_{T}^{\sin \left(2 \phi+\phi_{S}\right)}$ relates to the beam quark Boer-Mulders $h_{1}^{\perp}$ and the target quark pretzelosity $h_{1 T}^{\perp}$ and $A_{T}^{\sin \left(2 \phi-\phi_{S}\right)}$ relates to the beam quark Boer-Mulders $h_{1}^{\perp}$ and the target quark transversity function $h_{1}$.

\footnotetext{
ae-mail: marcia@lip.pt
} 


\section{Experimental setup}

COMPASS is a fixed target experiment located at CERN at the end of the M2 beam line extracted from the SPS. The experimental setup consists in a two stage spectrometer, for small and large angle particles, covering a wide angular and momentum range. Each spectrometer has a large number of tracking detectors as well as calorimeters and hodoscopes. A complete description can be found in [1]. In the DY setup for polarised muon pair measurement, the target consists in two target cells filled with ammonia and oppositely polarised transversely to the beam direction. The beam is negative pions with $190 \mathrm{GeV} / c$ momentum. Since DY has a very low cross-section it is mandatory to have high luminosity. Thus a beam intensity of $10^{8}$ pions/s will be used. In addition to the COMPASS standard setup a hadron absorber is placed downstream of the target to filter the hadrons and with a beam plug in its centre to stop the non-interacting beam. This hadron absorber degrades the resolutions of the measured kinematical quantities because it introduces multiple scattering on the muons. Regarding this issue a new scintillating fibres vertex detector is introduced in the beginning part of the absorber, as is shown in figure 1.

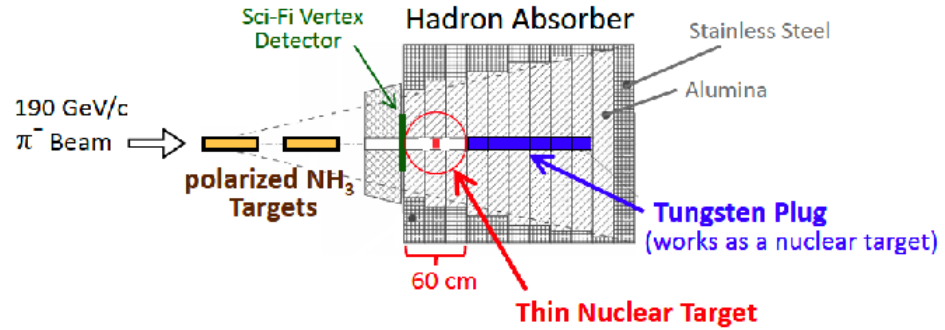

Figure 1. Sketch of the hadron absorber. In blue is visible the beam plug, in red the Al nuclear target and in green the vertex detector.

A thin aluminium target is placed $30 \mathrm{~cm}$ upstream of the tungsten beam plug. These two materials give us the opportunity to perform unpolarised DY studies, namely the study of the flavour dependence of the EMC effect [2].

\section{COMPASS: projections and prior results}

The experimental confirmation of the theoretical prediction that the Sivers and Boer-Mulders functions sign must change when accessed from DY or from SIDIS is considered a crucial test of the QCD TMD approach [3]. The Sivers asymmetry was already measured with SIDIS at COMPASS and the published results are presented in figure 2 together with the HERMES ones. For negative hadrons the asymmetry is zero. For positive hadrons the asymmetry is positive and slightly different between the two experiments. Notice that the $Q^{2}$ coverage is different between them, for $x>0.032,\left\langle Q^{2}\right\rangle=8.7$ $\mathrm{GeV} / c$ for COMPASS and $\left\langle Q^{2}\right\rangle=2.4 \mathrm{GeV} / c$ for HERMES.

Figure 3 shows the phase space coverage between SIDIS and DY processes in COMPASS. This must be taken into account to compare the extracted TMDs. Recently the SIDIS analysis was performed in different $Q^{2}$ bins, being one of them $Q^{2}>16(\mathrm{GeV} / c)^{2}$. The measured Sivers asymmetry in this bin is compatible with the result from figure 2 , the statistical accuracy being $\approx 1 \%$, the same as expected for Sivers from DY.

In 2009 a three-day beam test was done which proved the feasibility of the experiment. The target was made of two cells of polyethylene and a negative pion beam at $190 \mathrm{GeV} / c$ with an intensity of $1.5 \times 10^{7} \pi / \mathrm{s}$ was used. A hadron absorber prototype and a trigger based on calorimeter signals was also used. In the COMPASS DY experiment, a dimuon trigger based on hodoscopes with a high 


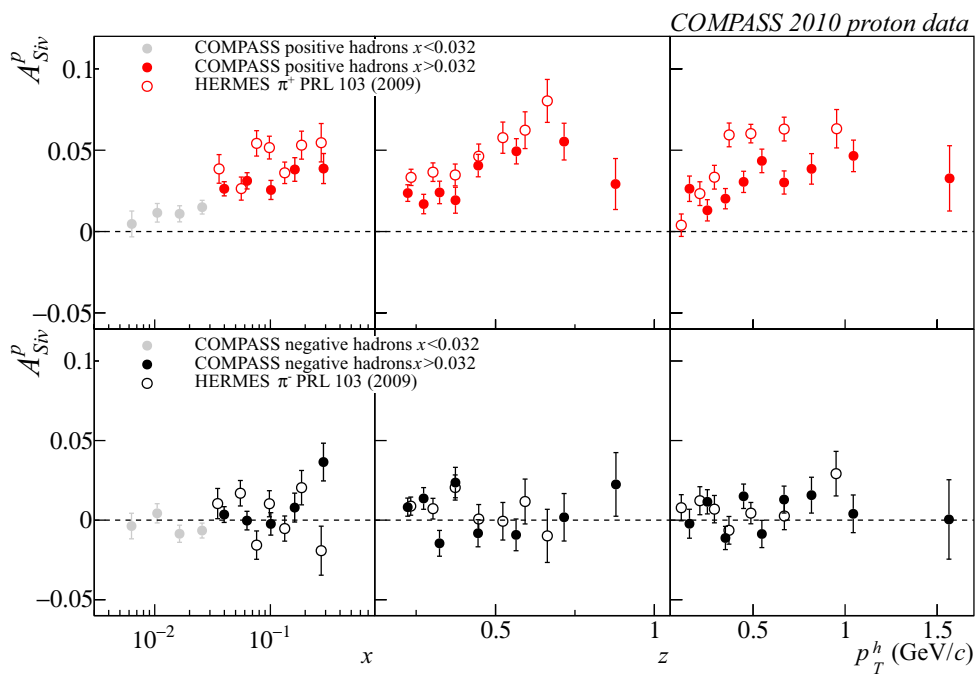

Figure 2. COMPASS SIDIS 2010 proton data. Sivers asymmetry for positive (red marks) and negative (black marks) hadrons together with the HERMES results published in 2009 [4].

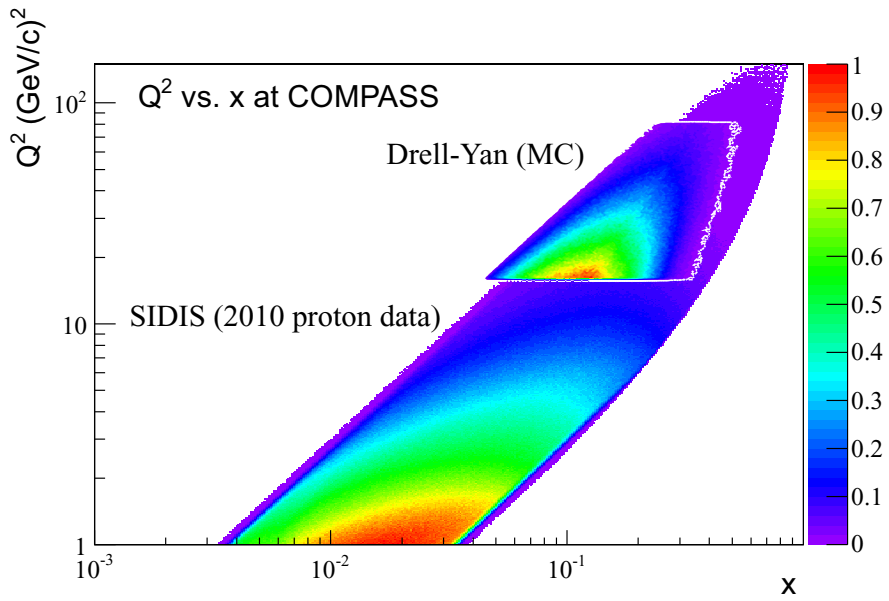

Figure 3. $Q^{2}$ versus $x$ phase space coverage at COMPASS.

Superposition of the SIDIS 2010 proton data with the DY MC simulation.

efficiency, purity and target pointing capability will be used. Some results from the analysis of the 2009 beam test are presented in figure 4.

The analysis confirmed the expectations concerning $J / \psi$ yields, given the low trigger efficiencies at the time. The $J / \psi$ pole and the mass resolution are in agreement with the MC simulations corresponding to this particular setup test. As visible in figure 4 the two target cells and the beam plug are distinguishable even if the absorber was not ideal. In the foreseen DY measurement a better $Z$ vertex resolution will be obtained, thanks to the new optimised absorber and the addition of the vertex detector.

\section{Event rates and statistical accuracy}

In the polarised DY measurement, the event rate in the mass region $4<M_{\mu \mu}<9 \mathrm{GeV} / c^{2}$ shall be 2000 events/day. Being one year of data taking approximately 140 days, 285000 events are expected. 

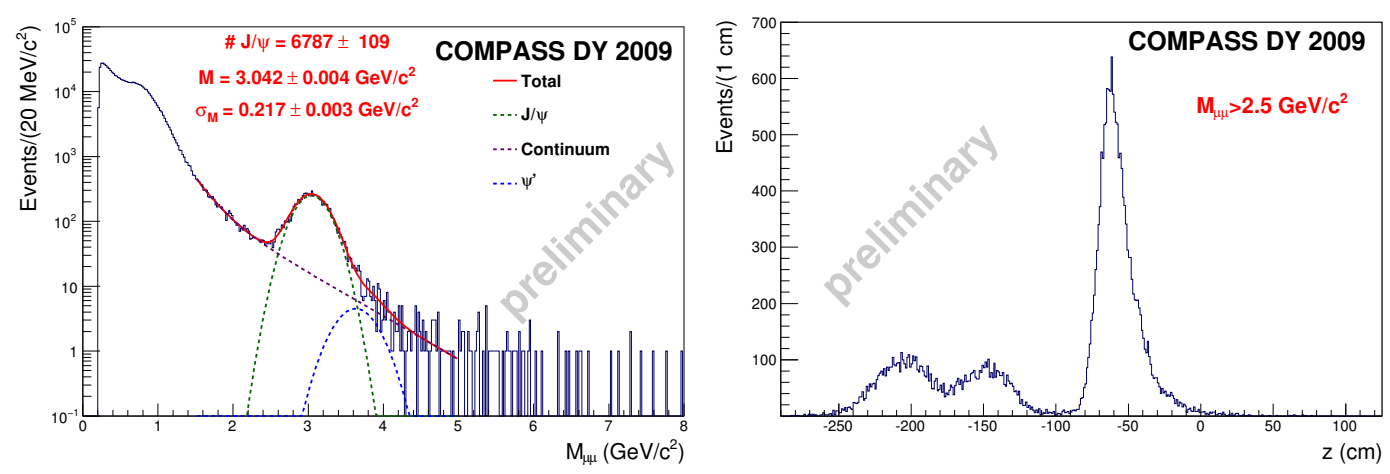

Figure 4. The dimuon mass distribution (left) and the $Z$ vertex distribution (right).

For such rate the expected statistical errors of the asymmetries are $\delta A_{U U}^{\cos 2 \phi}=0.5 \%, \delta A_{U T}^{\sin \phi_{S}}=1.3 \%$ and $\delta A_{U T}^{\sin \left(2 \phi+\phi_{S}\right)}=\delta A_{U T}^{\sin \left(2 \phi-\phi_{S}\right)}=2.7 \%$.

\section{Outlook}

A DY pilot run will start in October 2014 and will last for 2 months. A transversely polarised target made of two ammonia cells will be used. The beam will be negative pions at $190 \mathrm{GeV} / c$ with an intensity of $10^{8}$ pions/s. This will be the opportunity to tune and fix everything before the next year's physics data taking, which will provide the first ever DY polarised data. The aim is to contribute to the TMD PDFs knowledge, namely checking the Sivers sign change between DY and SIDIS results. The addition of the $\mathrm{Al}$ nuclear target and the presence of the $\mathrm{W}$ beam plug gives the opportunity to perform some studies of unpolarised DY data. After 2018 the possibility to have a second year of DY data taking is being discussed, which would allow for differential studies, as well as to address other interesting physics topics that require larger statistics.

\section{Acknowledgements}

This work was partially supported by the Portuguese Fundação para a Ciência e a Tecnologia.

\section{References}

[1] P. Abbon et al. [COMPASS Coll.], Nucl. Inst. Meth. A577, 455 (2007).

[2] D. Dutta et al., Phys. Rev. C83, 042201 (R) (2011)

[3] J.C. Collins, Phys. Lett. B536, 43 (2002).

[4] C. Adolph et al. [COMPASS Coll.], Phys. Lett. B717, 10 (2012). 